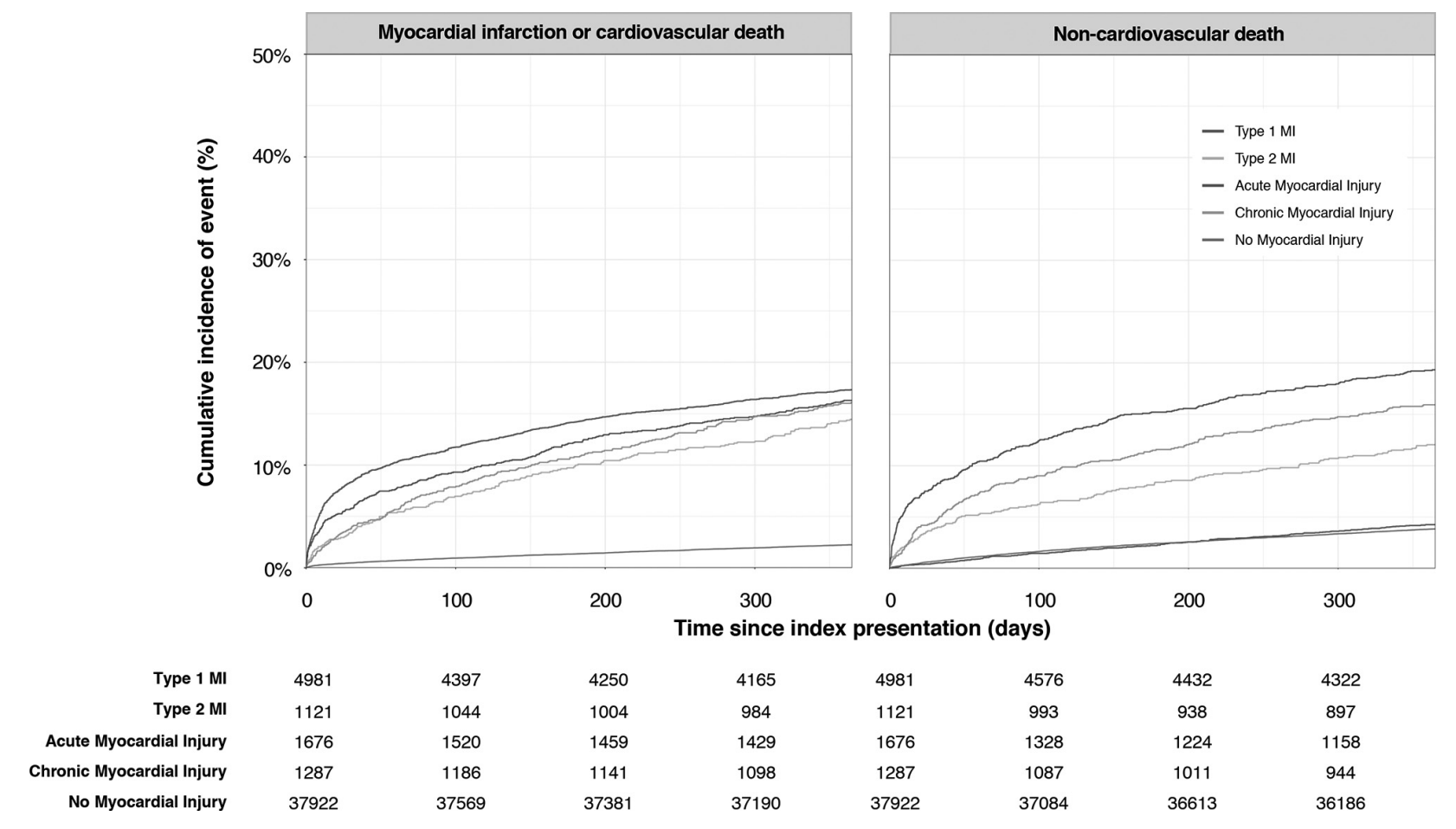

Abstract 144 Figure 2

\section{THE ROLE OF HIGH-SENSITIVITY C-REACTIVE PROTEIN IN PREDICTING MORTALITY BEYOND TROPONIN IN OVER 100,000 PATIENTS WITH SUSPECTED ACUTE CORONARY SYNDROME (NIHR HEALTH INFORMATICS COLLABORATIVE CRP-RISK STUDY)}

\begin{abstract}
${ }^{1}$ Amit Kaura*, 'Adam Hartley, 'Vasileios Panoulas, 'Ben Glampson, ${ }^{2} \mathrm{Jim}$ Davies, ${ }^{1}$ Abdulrahim Mulla, ${ }^{2}$ Kerrie Woods, ${ }^{3}$ Joe Omigie, ${ }^{4}$ Anoop D Shah, ${ }^{2}$ Keith Channon, ${ }^{1} J o n a t h a n N$ Weber, ${ }^{1}$ Mark R Thursz, ${ }^{1}$ Paul Elliott, ${ }^{4}$ Harry Hemingway, ${ }^{4}$ Bryan Williams, ${ }^{4}$ Folkert Asselbergs, ${ }^{5}$ Michael O'Sullivan, ${ }^{1}$ Dorian Haskard, ${ }^{6}$ Graham Lord, ${ }^{3}$ Narbeh Melikian, ${ }^{1}$ Daryl Francis, ${ }^{7}$ Wolfgang Koenig, ${ }^{8}$ Divaka Perera, ${ }^{3}$ Ajay Shah, ${ }^{3}$ Rajesh Kharbanda, ${ }^{4}$ Riyaz Patel, ${ }^{1}$ Jamil Mayet, ${ }^{1}$ Ramzi Khamis. ${ }^{1}$ NIHR Imperial College London Biomedical Research Centre and Imperial College Healthcare NHS Trust; ${ }^{2}$ NIHR University of Oxford BRC and Oxford University Hospitals NHS Foundation Trust; ${ }^{3}$ NIHR King's College London BRC and King's College Hospital NHS Foundation Trust; ${ }^{4}$ NIHR University College London BRC and University College London Hospitals NHS Foundation Trust; ${ }^{5}$ NIHR University of Cambridge BRC and Cambridge University Hospitals NHS Foundation Trust; ${ }^{6}$ NIHR King's College London BRC and Guy's and St Thomas' Hospital NHS Foundation Trust; ${ }^{7}$ Deutsches Herzzentrum München, Technische Universität München and DZHK; ${ }^{23}$ King's College London
\end{abstract}

\subsection{6/heartjnl-2019-BCS.142}

Background The incremental long-term prognostic value of high-sensitivity C-reactive protein (hsCRP) above troponin in a large real-world cohort of unselected patients presenting with suspected acute coronary syndromes (ACS) is unknown. We hypothesised that a mildly elevated hsCRP is associated with mortality risk in patients with suspected ACS, independent of troponin level.

Methods We used the National Institute for Health Research Health Informatics Collaborative data of 257,948 patients who had a troponin measured at 5 cardiac centres. We excluded patients with clinically abnormal white cell counts and hsCRP $>15 \mathrm{mg} / \mathrm{L}$ to try limiting the population to those without overt infections, malignancies or systemic inflammatory conditions that may confound our analyses. Patients were divided into four hsCRP groups $(<2,2-4.9,5-9.9$ and $10-15$ $\mathrm{mg} / \mathrm{L}$ ) and the association between hsCRP levels and all-cause mortality assessed.

Results There were 102,337 patients included in the analysis (hsCRP <2 mg/L ( $\mathrm{n}=38,390), 2-4.9 \mathrm{mg} / \mathrm{L} \quad(\mathrm{n}=27,397)$, 5-9.9 $\mathrm{mg} / \mathrm{L}(\mathrm{n}=26,957)$ and $10-15 \mathrm{mg} / \mathrm{L}(\mathrm{n}=9,593))$. figure $1 \mathrm{~A}$ displays cumulative mortality per hsCRP group, revealing increasing mortality with each consecutive group. figure 1B further stratifies the groups according to dichotomised peak troponin level as positive or negative. This shows the greatest mortality for patients in the highest hsCRP group who also had a positive troponin assay $(36.0 \%$ at 3 years).

In Cox regression analysis with time-dependent covariates, even mildly raised hsCRP was an independent predictor of mortality over time, after adjusting for age, gender, haemoglobin, white cell count, platelet count, creatinine and troponin positivity. There was a positive and graded relationship between hsCRP level and mortality at baseline, which remained at 3-years (hazard ratio $(95 \%$ CI) of 1.32 (1.181.48) for those with hsCRP $2.0-4.9 \mathrm{mg} / \mathrm{L}$, and $1.40(1.26-$ 1.57), and $2.00(1.75-2.28)$ for those with hsCRP $5-9.9 \mathrm{mg} / \mathrm{L}$ and $10-15 \mathrm{mg} / \mathrm{L}$, respectively.

We explored whether inclusion of hsCRP could better reclassify the population into at-risk mortality groups. The association with 30-day, 1-year and 3-year mortality was assessed using three different risk models (model 1: age, gender, haemoglobin, creatinine; model 2: model 1 plus troponin (positivity versus negativity); model 3: model 2 plus hsCRP groups. For cumulative mortality at each time point, each 
A

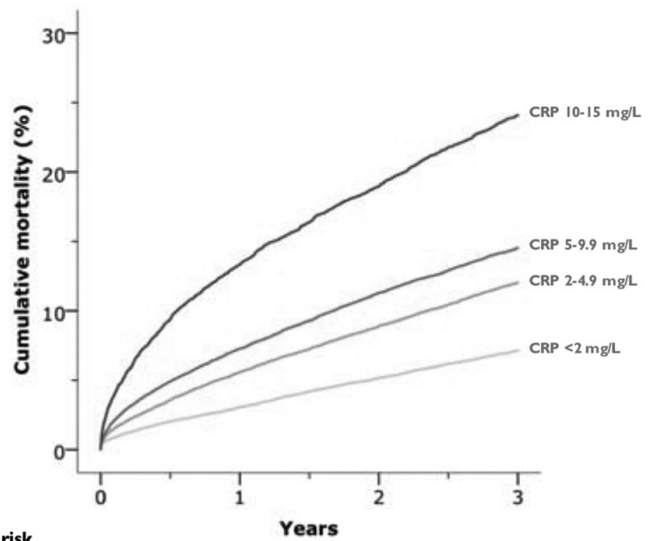

No. at risk

CRP $<2$

CRP 2-4.9

CRP 5-9.9

CRP 10-15

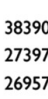

26957

9593

$\begin{array}{ccc}34086 & 28898 & 22311 \\ 23910 & 20096 & 15876 \\ 23860 & 21076 & 18140 \\ 7804 & 6493 & 5057\end{array}$

B

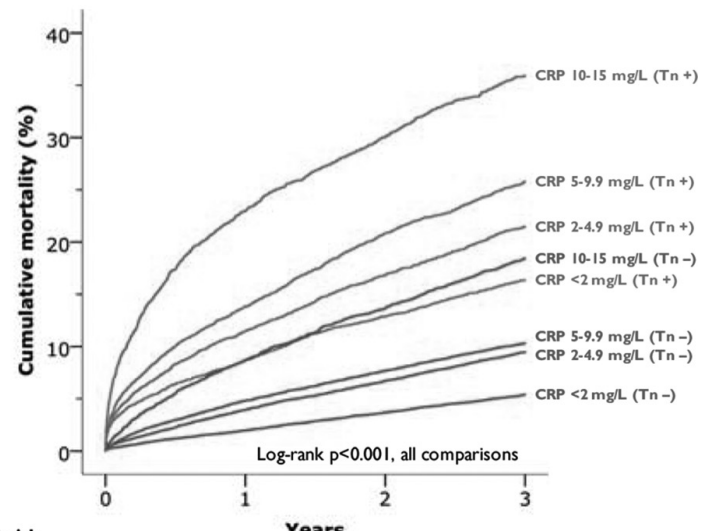

No. at risk

Troponin positive

CRP $<2 \quad 6099$

CRP 2-4.9 $\quad 5937$

CRP 5-9.9 $\quad 7402$

CRP 10-15 3126

Troponin negative

CRP $<2 \quad 32291$

CRP 2-4.9 21460

CRP 5-9.9 19555

CRP 10-15 $\quad 6467$

Abstract 145 Figure 1 Kaplan-Meier mortality curve by (A) hsCRP level and (B) hsCRP level and troponin positivity

successive model was better able to discriminate risk than its precursor $(\mathrm{p}<0.0001)$; such that inclusion of troponin and hsCRP gave the most robust risk discrimination. Model 3 achieved an AUROC $>0.8$ at 30 days, 1-year and 3-year mortality, surpassing the use of troponin on its own.

Conclusion These multi-centre, real-world data from a large cohort of patients with suspected ACS identify hsCRP as a clinically meaningful prognostic marker in addition to troponin levels and point to its potential utility in selecting patients for novel treatments targeting inflammation.

Conflict of Interest No conflicts of interest

\section{THE PROGNOSTIC IMPLICATION OF A POSITIVE TROPONIN ACROSS THE AGE SPECTRUM IN A QUARTER OF A MILLION PATIENTS WITH SUSPECTED ACUTE CORONARY SYNDROME (NIHR HEALTH INFORMATICS COLLABORATIVE TROP-RISK STUDY)}

${ }^{1}$ Amit Kaura*, ${ }^{1}$ Vasileios Panoulas, ${ }^{1}$ Ben Glampson, ${ }^{2} \mathrm{Jim}$ Davies, ${ }^{1}$ Abdulrahim Mulla,

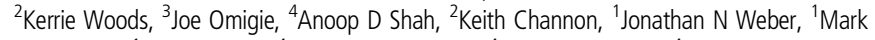
$\mathrm{R}$ Thursz, ${ }^{1}$ Paul Elliott, ${ }^{4}$ Harry Hemingway, ${ }^{4}$ Bryan Williams, ${ }^{4}$ Folkert Asselbergs, ${ }^{5}$ Michael O'Sullivan, ${ }^{6}$ Graham Lord, ${ }^{3}$ Narbeh Melikian, ${ }^{2}$ Rajesh Kharbanda, ${ }^{3}$ Ajay Shah,

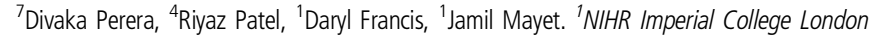
Biomedical Research Centre and Imperial College Healthcare NHS Trust; ${ }^{2}$ NIHR University of Oxford BRC and Oxford University Hospitals NHS Foundation Trust; ${ }^{3}$ NIHR King's College London BRC and King's College Hospital NHS Foundation Trust; ${ }^{4}$ NIHR University College London BRC and University College London Hospitals NHS Foundation Trust; ${ }^{5}$ NIHR University of Cambridge BRC and Cambridge University Hospitals NHS Foundation Trust; ${ }^{6}$ NIHR King's College London BRC and Guy's and St Thomas' Hospital NHS Foundation Trust; ${ }^{7}$ King's College London

10.1136/heartjnl-2019-BCS.143

Background In the past two decades, assays for troponin have undergone vast improvements, allowing fast detection of troponin with increased precision. With improved sensitivity of current troponin assays, more patients end up with a positive troponin result. There is limited data to help inform the implications of a positive troponin test across the age spectrum, in clinical practice. The aim of this study was to investigate the overall prognostic impact of a positive troponin result on all-cause mortality in patients in whom troponin testing has been done for clinical purposes.

Methods The NIHR Health Informatics Collaborative (NHIC) project was established to enable the sharing and repurposing of routinely captured clinical data for re-use in research. All troponin values measured during the study period (generally 2010 to 2017) were assembled from five contributing cardiovascular centres. The results were dichotomised as being positive or negative based on the 99th percentile of the upper limit of normal for all relevant troponin assays. All patients were followed up on the National Health Service Spine Application, Summary Care Record until death or censoring on 1st April 2017. Statistical analyses were performed using SPSS software version 24.0 (SPSS Inc., Chicago, Illinois, United States).

Results 257948 patients underwent troponin assessment during the study period. The median age was 65 (IQR 50-79) and $55.3 \%$ were men. During a median follow-up of 1198 (IQR 514-1866) days, there were 55850 (21.7\%) deaths. The proportion of troponins that were positive progressively rose with age from $9.1 \%$ in the $18-29$ band to $50.0 \%$ in the over 90 s.

The median positive troponin was 2280 times higher than the median negative troponin, and this relationship was largely invariant with age. A positive troponin was associated with an overall 3.2-fold higher mortality hazard (95\% CI 3.1-3.2) than a negative troponin over 3 years. For young patients (18-29 years) this was a particularly strong effect, with a mortality hazard ratio of 10.6 (95\% CI $8.5-13.3)$. The effect declined progressively with age to a mortality hazard of 1.5 - 